\title{
Generalized Stern polynomials and hyperbinary representations
}

by

\author{
Karl DILCHER and Larry ERICKSEN
}

Presented by Andrzej SCHINZEL

Summary. We use two different but related types of generalized Stern polynomials, recently introduced by the authors, to give complete characterizations of all hyperbinary expansions of a given positive integer. We also derive explicit formulas for these generalized Stern polynomials and use them to establish further characterizations of hyperbinary expansions, using binomial coefficients. We then introduce a 2-parameter analogue of the two types of polynomials, which leads to more explicit versions of earlier results. Finally, we explore further generalizations of the polynomials studied in this paper.

1. Introduction. The Stern sequence, also known as Stern's diatomic sequence, is one of the most remarkable integer sequences in number theory and combinatorics. Using the notation $(a(n))_{n \geq 0}$, it can be defined by $a(0)=0, a(1)=1$, and for $n \geq 1$ by

$$
\begin{aligned}
a(2 n) & =a(n), \\
a(2 n+1) & =a(n)+a(n+1) .
\end{aligned}
$$

Numerous properties and references can be found, e.g., in [2], [13, A002487], or [14]. This integer sequence was independently extended to two different concepts of Stern polynomials: one type was introduced in [8], and another type was defined in [11] and further studied in [9, 16, 17, 18, 20, 21]. More recently, the present authors extended these two polynomial sequences as follows (see [6, 7]).

2010 Mathematics Subject Classification: Primary 05A15; Secondary 11B83.

Key words and phrases: Stern sequence, Stern polynomials, hyperbinary expansions, generating functions.

Received 19 September 2016.

Published online 17 March 2017. 
DeFinition 1.1. Let $t$ be a fixed positive integer parameter.

(a) The type-1 generalized Stern polynomials $a_{1, t}(n ; z)$ are polynomials in $z$ defined by $a_{1, t}(0 ; z)=0, a_{1, t}(1 ; z)=1$, and for $n \geq 1$ by

$$
\begin{aligned}
a_{1, t}(2 n ; z) & =z a_{1, t}\left(n ; z^{t}\right), \\
a_{1, t}(2 n+1 ; z) & =a_{1, t}\left(n ; z^{t}\right)+a_{1, t}\left(n+1 ; z^{t}\right) .
\end{aligned}
$$

(b) The type-2 generalized Stern polynomials $a_{2, t}(n ; z)$ are polynomials in $z$ defined by $a_{2, t}(0 ; z)=0, a_{2, t}(1 ; z)=1$, and for $n \geq 1$ by

$$
\begin{aligned}
a_{2, t}(2 n ; z) & =a_{2, t}\left(n ; z^{t}\right), \\
a_{2, t}(2 n+1 ; z) & =z a_{2, t}\left(n ; z^{t}\right)+a_{2, t}\left(n+1 ; z^{t}\right) .
\end{aligned}
$$

See Table 1 for the first 16 of each of these polynomials. By comparing (1.2) - 1.5 with (1.1), we see that for $z=1$ both sequences reduce to Stern's diatomic sequence, i.e.,

$$
a_{1, t}(n ; 1)=a_{2, t}(n ; 1)=a(n) \quad(t \geq 1, n \geq 0) .
$$

Furthermore, the sequences $a_{1,1}(n ; z)$ and $a_{2,2}(n ; z)$ are the Stern polynomials introduced in [11] and in [8], respectively.

Table 1. $a_{1, t}(n ; z)$ and $a_{2, t}(n ; z), 1 \leq n \leq 16$

\begin{tabular}{rll}
\hline$n$ & $a_{1, t}(n ; z)$ & $a_{2, t}(n ; z)$ \\
\hline 1 & 1 & 1 \\
2 & $z$ & 1 \\
3 & $1+z^{t}$ & $1+z$ \\
4 & $z^{t+1}$ & 1 \\
5 & $1+z^{t}+z^{t^{2}}$ & $1+z+z^{t}$ \\
6 & $z+z^{t^{2}+1}$ & $1+z^{t}$ \\
7 & $1+z^{t^{2}}+z^{t^{2}+t}$ & $1+z+z^{t+1}$ \\
8 & $z^{t^{2}+t+1}$ & 1 \\
9 & $1+z^{t^{2}}+z^{t^{2}+t}+z^{t^{3}}$ & $1+z+z^{t}+z^{t^{2}}$ \\
10 & $z+z^{t^{2}+1}+z^{t^{3}+1}$ & $1+z^{t}+z^{t^{2}}$ \\
11 & $1+z^{t}+z^{t^{2}}+z^{t^{3}}+z^{t^{3}+t}$ & $1+z+z^{t+1}+z^{t^{2}}+z^{t^{2}+1}$ \\
12 & $z^{t+1}+z^{t^{3}+t+1}$ & $1+z^{t^{2}}$ \\
13 & $1+z^{t}+z^{t^{3}}+z^{t^{3}+t}+z^{t^{3}+t^{2}}$ & $1+z+z^{t}+z^{t^{2}+1}+z^{t^{2}+t}$ \\
14 & $z+z^{t^{3}+1}+z^{t^{3}+t^{2}+1}$ & $1+z^{t}+z^{t^{2}+t}$ \\
15 & $1+z^{t^{3}}+z^{t^{3}+t^{2}}+z^{t^{3}+t^{2}+t}$ & $1+z+z^{t+1}+z^{t^{2}+t+1}$ \\
16 & $z^{t^{3}+t^{2}+t+1}$ & 1 \\
\hline
\end{tabular}

Table 1 indicates that both sequences of polynomials have a special structure. While it is easily seen that for $t=1$ the exponents in a given polynomial can coincide, for $t \geq 2$ the situation is quite different. 
TheOREM 1.1 ([5], [7]). For integers $t \geq 2$ and $n \geq 0$, the coefficients of $a_{1, t}(n ; z)$ and $a_{2, t}(n ; z)$ are only 0 or 1 . Furthermore, all exponents of $z$ are polynomials in $t$ with only 0 or 1 as coefficients.

This result and (1.6) show that the number of terms of both polynomials is given by the Stern number $a(n)$.

Certain subsequences of both $a_{1, t}(n ; z)$ and $a_{2, t}(n ; z)$ were recently applied by the authors in two different settings, namely tilings, colorings and lattice paths [6], and continued fractions [7]. This paper, however, along with a previous study [5], is concerned with a combinatorial object that has long been connected with Stern's diatomic sequence, namely the concept of hyperbinary representations or expansions.

A hyperbinary expansion of an integer $n \geq 1$ is an expansion of $n$ as a sum of powers of 2 , each power being used at most twice.

ExAMPLE 1. The hyperbinary expansions of $n=10$ are

$$
8+2, \quad 8+1+1, \quad 4+4+2, \quad 4+4+1+1, \quad 4+2+2+1+1,
$$

an example we are going to use throughout much of this paper. One of the hyperbinary expansions is always the unique binary expansion, e.g., $8+2$ among the expansions in (1.7).

Within the framework of a more general study of binary partition functions, Reznick [14, Theorem 5.2] proved the following fundamental result.

THEOREM 1.2 ([14]). The number of hyperbinary expansions of an integer $n \geq 1$ is given by the Stern number $a(n+1)$.

As an example of this result, consider $n=10$. We have $a(11)=5$ (see Table 1), which is consistent with Example 1.

It is the main purpose of this paper to extend Theorem 1.2 in several directions, thus also extending other known results. In Section 2 we will see that both types of the generalized Stern polynomials can be used to characterize all $a(n+1)$ hyperbinary expansions of $n$ in two different ways. In Section 3 we use a known connection, due to Carlitz, between the Stern sequence and certain binomial coefficients as a point of departure to prove explicit formulas for both types of generalized Stern polynomials. These formulas will then be used to derive two different but related characterizations of hyperbinary expansions, using binomial coefficients. In Section 4 we define a 2-parameter extension of both types of generalized Stern polynomials, which gives rise to an even more explicit characterization of hyperbinary expansions. We conclude this paper with some additional remarks in Section 5 . 
2. Two views of hyperbinary expansions. In this section we will see that Reznick's Theorem 1.2 can be extended in two different but related directions, and this is where both types of generalized Stern polynomials enter in their full generality. We begin by quoting some known results.

2.1. Counting repeated powers of 2. Reznick's theorem was first refined by Bates and Mansour [1] and independently by Stanley and Wilf [19]. They proved results that are equivalent to the following version, as given in [5], where the type-2 generalized Stern polynomials are used.

Theorem 2.1 ([1], [19]). Given an integer $n \geq 1$, write

$$
a_{2,1}(n+1 ; z)=\sum_{j \geq 0} c_{n, j} z^{j} .
$$

Then for each $j \geq 0, c_{n, j}$ is the number of hyperbinary expansions of $n$ that have exactly $j$ repeated powers of 2 .

EXAMPle 2. Consider again $n=10$, with the hyperbinary expansions (1.7). Since binary expansions are unique, we always have $c_{n, 0}=1$. Further, $8+1+1$ and $4+4+2$ have exactly one repeated power of 2 , so $c_{10,1}=2$. Next, $4+4+1+1$ and $4+2+2+1+1$ have exactly two repeated powers of 2 , and thus $c_{10,2}=2$. So finally, the right-hand side of $(2.1)$ is $1+2 z+2 z^{2}$, which according to Table 1 is indeed $a_{2,1}(11 ; z)$.

Using the structure of the generalized Stern polynomials $a_{2, t}(n ; z)$ (see Theorem 1.1), we can further refine Theorem 2.1 by giving complete characterizations of all hyperbinary expansions of a given integer $n \geq 1$.

Theorem $2.2([5])$. Given an integer $n \geq 1$, let $\mathcal{P}_{2}(n+1)$ be the set of exponents of $z$ in $a_{2, t}(n+1 ; z)$, i.e.,

$$
a_{2, t}(n+1 ; z)=\sum_{p \in \mathcal{P}_{2}(n+1)} z^{p(t)}
$$

Then each hyperbinary expansion of $n$ corresponds to exactly one polynomial in $\mathcal{P}_{2}(n+1)$, as follows: if

$$
p(t)=t^{\alpha_{1}}+\cdots+t^{\alpha_{r}} \in \mathcal{P}_{2}(n+1), \quad r \geq 0,0 \leq \alpha_{1}<\cdots<\alpha_{r},
$$

then exactly the powers $2^{\alpha_{1}}, \ldots, 2^{\alpha_{r}}$ are repeated in the expansion. Here $r=0$ corresponds to the zero polynomial, and thus to the case of no repeated powers, i.e., the unique binary expansion.

Example 3 . We continue with the case $n=10$. Table 1 gives us

$$
\mathcal{P}_{2}(11)=\left\{0,1,1+t, t^{2}, 1+t^{2}\right\} .
$$

Accordingly, the five hyperbinary expansions are characterized by the following repeated powers of 2 : 
- none, so $10=2+8$;

- $2^{0}$, so $10=\mathbf{1}+\mathbf{1}+8$;

- $2^{0}$ and $2^{1}$, so $10=\mathbf{1}+\mathbf{1}+\mathbf{2}+\mathbf{2}+4$;

- $2^{2}$, so $10=2+\mathbf{4}+\mathbf{4}$;

- $2^{0}$ and $2^{2}$, so $10=\mathbf{1}+\mathbf{1}+\mathbf{4}+\mathbf{4}$.

2.2. Counting non-repeated powers of 2. Early on in [11], Klavžar et al. used their newly defined Stern polynomials to prove what amounts to a close analogue to Theorem 2.1. In fact, their result predates Theorem 2.1 by a few years. Using our notation, it can be stated as follows.

THEOREM 2.3 ([1]). Given an integer $n \geq 1$, write

$$
a_{1,1}(n+1 ; z)=\sum_{j \geq 0} d_{n, j} z^{j} .
$$

Then for each $j \geq 0, d_{n, j}$ is the number of hyperbinary expansions of $n$ that have exactly $j$ non-repeated powers of 2 .

Just as Theorem 2.2 is a refinement of Theorem 2.1, we will now prove an analogous refinement of Theorem 2.3. This is the first new result in this paper.

THEOREM 2.4. Given an integer $n \geq 1$, let $\mathcal{P}_{1}(n+1)$ be the set of exponents of $z$ in $a_{1, t}(n+1 ; z)$, i.e.,

$$
a_{1, t}(n+1 ; z)=\sum_{p \in \mathcal{P}_{1}(n+1)} z^{p(t)} .
$$

Then each hyperbinary expansion of $n$ corresponds to exactly one polynomial in $\mathcal{P}_{1}(n+1)$, as follows: if

$$
p(t)=t^{\alpha_{1}}+\cdots+t^{\alpha_{r}} \in \mathcal{P}_{1}(n+1), \quad r \geq 0,0 \leq \alpha_{1}<\cdots<\alpha_{r},
$$

then exactly the powers $2^{\alpha_{1}}, \ldots, 2^{\alpha_{r}}$ are not repeated in the expansion. Here $r=0$ corresponds to the zero polynomial, and thus to the case of all powers being repeated, that is, the unique case given by the binary expansion of $n / 2$, when $n$ is even.

Before proving this result, we illustrate it with our standard example.

Example 4. Continuing with $n=10$, we note that Table 1 gives us

$$
\mathcal{P}_{1}(11)=\left\{0, t, t^{2}, t^{3}, t+t^{3}\right\} .
$$

The five hyperbinary expansions are then characterized by the non-repeated powers of 2 as follows:

- none, so $10=1+1+4+4$;

- $2^{1}$, so $10=\mathbf{2}+4+4$;

- $2^{2}$, so $10=1+1+2+2+4$;

- $2^{3}$, so $10=1+1+\mathbf{8}$;

- $2^{1}$ and $2^{3}$, so $10=\mathbf{2}+\mathbf{8}$. 
Proof of Theorem 2.4. We prove this by induction on $n$. For $n=1$, the only hyperbinary expansion (HBE) is 1 itself. On the other hand, $a_{1, t}(2 ; z)$ $=z$, so $\mathcal{P}_{1}(2)=\left\{t^{0}\right\}$, indicating that $2^{0}=1$ is not repeated, as required.

For $n=2$, we have the two HBEs 2 and $1+1$, while on the other hand $a_{1, t}(3 ; z)=1+z^{t}$, so $\mathcal{P}_{1}(3)=\left\{0, t^{1}\right\}$. This indicates that one HBE has no non-repeated powers of 2 and the other has $2^{1}$ as only non-repeated power, which is again as required.

For the induction step we assume that the statement of Theorem 2.4 holds up to some $n-1 \geq 2$. We distinguish between two cases.

(a) Suppose that $n$ is odd, say $n=2 k+1$. Then each HBE of $n$ contains exactly one copy of $2^{0}$, and we assume that in a given HBE of $n$ the powers

$$
2^{0}, 2^{\alpha_{2}}, \ldots, 2^{\alpha_{r}}, \quad r \geq 1,0<\alpha_{2}<\cdots<\alpha_{r},
$$

are not repeated, where $r=1$ means that only $2^{0}$ is not repeated. Now subtract the part $2^{0}$ from each HBE of $n$ and divide by 2 . Then the HBE of $n$ with the property (2.5) corresponds to the HBE of $\frac{1}{2}(n-1)=k$ where the powers

$$
2^{\alpha_{2}-1}, \ldots, 2^{\alpha_{r}-1}, \quad r \geq 1,0<\alpha_{2}<\cdots<\alpha_{r},
$$

are not repeated. By the induction hypothesis, we have

$$
a_{1, t}(k+1 ; z)=\sum_{p \in \mathcal{P}_{1}(k+1)} z^{p(t)}
$$

with

$$
p(t)=t^{\alpha_{2}-1}+\cdots+t^{\alpha_{r}-1}, \quad r \geq 1,0 \leq \alpha_{2}<\cdots<\alpha_{r},
$$

as element of the set $\mathcal{P}_{1}(k+1)$. Also note that the relationship between the HBEs of $n$ with property (2.5) and those of $k$ with property (2.6) is a bijection. On the other hand, by (1.2) with $n$ replaced by $k+1$ we have

$$
a_{1, t}(n+1 ; z)=a_{1, t}(2 k+2 ; z)=z a_{1, t}\left(k+1 ; z^{t}\right) .
$$

This means that each $p \in \mathcal{P}_{1}(k+1)$ of the form (2.7) corresponds to the exponent polynomial

$$
1+t^{\alpha_{2}}+\cdots+t^{\alpha_{r}} \in \mathcal{P}_{1}(n+1),
$$

and vice versa. This, in turn, corresponds to the HBE of $n$ with property (2.5), which completes the proof of this case.

(b) Suppose now that $n$ is even, say $n=2 k$. Then the HBEs of $n$ fall into two categories:

(i) Those that have no part $2^{0}$, and the powers

$$
2^{\alpha_{1}}, \ldots, 2^{\alpha_{r}}, \quad r \geq 0,0<\alpha_{2}<\cdots<\alpha_{r},
$$

are not repeated, where $r=0$ indicates that there are no nonrepeated powers of 2 . 
(ii) Those that have a repeated part $2^{0}$, and the powers

$$
2^{\beta_{1}}, \ldots, 2^{\beta_{s}}, \quad s \geq 0,0<\beta_{2}<\cdots<\beta_{s},
$$

are not repeated, with the case $s=0$ again indicating that there are no non-repeated powers of 2 .

Now, using the same argument as in part (a), we conclude that there is a oneto-one correspondence between all HBEs of $n$ in (i) and those of $n / 2=k$ with powers $2^{\alpha_{2}-1}, \ldots, 2^{\alpha_{r}-1}$ not repeated. Similarly, by removing the double $2^{0}$ from all HBEs of $n$ in (ii), we see that there is a one-to-one correspondence between these and the HBEs of $\frac{1}{2}(n-2)=k-1$ with powers $2^{\beta_{2}-1}, \ldots, 2^{\beta_{s}-1}$ not repeated.

Finally, by (1.3) with $n$ replaced by $k$, we have

$$
a_{1, t}(2 k+1 ; z)=a_{1, t}\left(k+1 ; z^{t}\right)+a_{1, t}\left(k ; z^{t}\right) .
$$

Using the same arguments as in part (a), along with the induction hypothesis, we see that the left-hand side of (2.8) represents all HBEs of $n=2 k$ with properties (i) and (ii). This completes the proof by induction.

3. Connections with binomial coefficients. In addition to the connection between hyperbinary expansions and Stern's diatomic sequence given in Theorem 1.2, there is also an interesting connection with binomial coefficients. The following result was proved by Carlitz [3, p. 17] in different notation and in connection with a wider study of certain polynomial sequences.

Theorem 3.1 ([3]). For a fixed $n \geq 0$, the number of odd binomial coefficients $\left(\begin{array}{c}n-k \\ k\end{array}\right)$ is given by the Stern number $a(n+1)$.

This can be rewritten by considering binomial coefficients modulo 2, as follows:

$$
\left(\begin{array}{l}
n \\
k
\end{array}\right)^{*} \equiv\left(\begin{array}{l}
n \\
k
\end{array}\right)(\bmod 2), \quad\left(\begin{array}{l}
n \\
k
\end{array}\right)^{*} \in\{0,1\} .
$$

Then Theorem 3.1 can be stated as

$$
a(n+1)=\sum_{k=0}^{\lfloor n / 2\rfloor}\left(\begin{array}{c}
n-k \\
k
\end{array}\right)^{*},
$$

which was earlier obtained in [10, p. 319]. We will now extend this identity in two different but related directions, as we did in Subsections 2.1 and 2.2.

3.1. Counting repeated powers of 2. A first polynomial analogue of the identity (3.1) was given in [5] as

$$
a_{2,2}(n+1 ; z)=\sum_{k=0}^{\lfloor n / 2\rfloor}\left(\begin{array}{c}
n-k \\
k
\end{array}\right)^{*} z^{k} .
$$


For a further extension to type-2 generalized Stern polynomials, and also for the next subsection, we require the following definition.

Definition 3.1. Let $n \geq 0$ be an integer, and let $n=\sum_{j \geq 0} c_{j} 2^{j}, c_{j} \in$ $\{0,1\}$, be the binary expansion of $n$. Then for integers $t \geq 1$ we define

$$
d_{t}(n):=\sum_{j \geq 0} c_{j} t^{j}
$$

Various small values of $d_{t}(n)$ can be found in [5, Table 2], with references to entries in the OEIS [13. In particular, $d_{1}(n)$ gives the binary weight or Hamming weight of $n$, obviously $d_{2}(n)=n$, and $d_{4}(n)$ is known as the Moser-de Bruijn sequence. Furthermore, the definition (3.3) immediately implies the identities

$$
d_{t}(2 n)=t d_{t}(n), \quad d_{t}(2 n+1)=t d_{t}(n)+1 .
$$

We can now quote the following extension of (3.1) and (3.2), which can also be seen as an explicit formula for the type-2 generalized Stern polynomials.

THEOREM $3.2([5])$. For integers $t \geq 1$ and $n \geq 0$ we have

$$
a_{2, t}(n+1 ; z)=\sum_{k=0}^{\lfloor n / 2\rfloor}\left(\begin{array}{c}
n-k \\
k
\end{array}\right)^{*} z^{d_{t}(k)} .
$$

This was then used in [5] to prove the following result, which again brings in the hyperbinary expansions.

Theorem 3.3 ([5]). Let $n \geq 2$ be an integer and denote by $k_{1}, \ldots, k_{\nu}$ those integers $0 \leq k \leq\lfloor n / 2\rfloor$ for which $\left(\begin{array}{c}n-k \\ k\end{array}\right)$ is odd. Then each hyperbinary expansion of $n$ corresponds to exactly one $k_{j}, j=1, \ldots, \nu$, as follows: the powers of 2 in the binary expansion of $k_{j}$ are exactly the repeated powers of 2 in a hyperbinary expansion of $n$.

EXAmple 5. Considering again $n=10$, we find that $\left(\begin{array}{c}10-k \\ k\end{array}\right)$ is odd for $k=0,1,3,4$, and 5 . Accordingly, the four proper hyperbinary expansions of $n=10$ are characterized as follows:

- $k_{1}=0$, so $10=8+2$;

- $k_{2}=1$, so $10=8+\mathbf{1}+\mathbf{1}$

- $k_{3}=3$, so $10=4+\mathbf{2}+\mathbf{2}+\mathbf{1}+\mathbf{1}$;

- $k_{4}=4$, so $10=4+4+2$;

- $k_{5}=5$, so $10=4+4+1+1$.

3.2. Counting non-repeated powers of 2. The second extension of (3.1), which provides an explicit formula for the type-1 generalized Stern polynomials, turns out to be remarkably similar in shape to its type- 2 analogue (3.5). Once again the class of sequences $d_{t}(n)$ is used. 
TheOREM 3.4. For integers $t \geq 1$ and $n \geq 0$ we have

$$
a_{1, t}(n+1 ; z)=\sum_{k=0}^{\lfloor n / 2\rfloor}\left(\begin{array}{c}
n-k \\
k
\end{array}\right)^{*} z^{d_{t}(n-2 k)} .
$$

Proof. The method of proof is similar to that of (3.5), given in [5]. In particular, we use the following identity, valid for non-negative integers $m, n$ and $a, b \in\{0,1\}$ :

$$
\left(\begin{array}{c}
2 m+a \\
2 n+b
\end{array}\right)^{*}= \begin{cases}0 & \text { when } a=0 \text { and } b=1 \\
\left(\begin{array}{l}
m \\
n
\end{array}\right)^{*} & \text { otherwise. }\end{cases}
$$

This identity follows from a well-known congruence of Lucas, but it was already used by Carlitz [3, p. 18f.]. We prove (3.6) by showing that both sides of the identity satisfy the same set of recurrence relations with the same initial conditions. We denote the right-hand side of $(3.6)$ by $\bar{a}_{t}(n+1 ; z)$. First, it is easy to verify that the initial conditions $\bar{a}_{t}(1 ; z)=1$ and $\bar{a}_{t}(2 ; z)=z$ hold, as required (see Table 1). Now we distinguish between two cases:

(i) Let $n$ be odd, say $n=2 m-1, m \geq 2$. Then by 3.7 we have

$$
\begin{aligned}
\bar{a}_{1, t}(2 m ; z) & =\sum_{k \geq 0}\left(\begin{array}{c}
2 m-1-k \\
k
\end{array}\right)^{*} z^{d_{t}(2 m-1-k)} \\
& =\sum_{j \geq 0}\left(\begin{array}{c}
m-1-j \\
j
\end{array}\right)^{*} z^{d_{t}(2 m-1-2 j)} .
\end{aligned}
$$

Now the second identity in 3.4 gives

$$
d_{t}(2 m-1-2 j)=d_{t}((2 m-2-2 j)+1)=t d_{t}(m-1-j)+1,
$$

and thus

$$
\bar{a}_{1, t}(2 m ; z)=\sum_{j \geq 0}\left(\begin{array}{c}
m-1-j \\
j
\end{array}\right)^{*}\left(z^{t}\right)^{d_{t}(m-1-j)} z=z \bar{a}_{1, t}\left(m ; z^{t}\right),
$$

which agrees with 1.2 .

(ii) Let $n$ be even, say $n=2 m, m \geq 1$. We split the summation index into odd and even $k$ :

$$
\begin{aligned}
\bar{a}_{1, t}(2 m+1 ; z) & =\sum_{k \geq 0}\left(\begin{array}{c}
2 m-k \\
k
\end{array}\right)^{*} z^{d_{t}(2 m-k)} \\
& =\sum_{j \geq 0}\left(\begin{array}{c}
2 m-2 j-1 \\
2 j+1
\end{array}\right)^{*} z^{d_{t}(2 m-2 j-1)}+\sum_{j \geq 0}\left(\begin{array}{c}
2 m-2 j \\
2 j
\end{array}\right)^{*} z^{d_{t}(2 m-2 j)} .
\end{aligned}
$$

Now we apply (3.7) to both binomial coefficients in the last line, and use (3.8) for the first exponent. For the second exponent, (3.4) gives $d_{t}(2 m-2 j)=$ $t d_{t}(m-j)$, and altogether we get 


$$
\begin{aligned}
\bar{a}_{1, t}(2 m+1 ; z) & =\sum_{j \geq 0}\left(\begin{array}{c}
m-1-j \\
j
\end{array}\right)^{*}\left(z^{t}\right)^{d_{t}(m-1-j)} z+\sum_{j \geq 0}\left(\begin{array}{c}
m-j \\
j
\end{array}\right)^{*}\left(z^{t}\right)^{d_{t}(m-j)} \\
& =z \bar{a}_{1, t}\left(m ; z^{t}\right)+\bar{a}_{1, t}\left(m+1 ; z^{t}\right),
\end{aligned}
$$

which agrees with (1.3). This completes the proof.

We are now ready to state and prove a close analogue of Theorem 3.3.

TheOrem 3.5. Let $n \geq 2$ be an integer and denote by $k_{1}, \ldots, k_{\nu}$ those integers $0 \leq k \leq\lfloor n / 2\rfloor$ for which $\left(\begin{array}{c}n-k \\ k\end{array}\right)$ is odd, and set $h_{j}:=n-2 k_{j}$, $j=1, \ldots, \nu$. Then each hyperbinary expansion of $n$ corresponds to exactly one $h_{j}, j=1, \ldots, \nu$, as follows: the powers of 2 in the binary expansion of $h_{j}$ are exactly the non-repeated powers of 2 in a hyperbinary expansion of $n$.

EXAMPLE 6. Considering again $n=10$, we recall that $\left(\begin{array}{c}10-k \\ k\end{array}\right)$ is odd for $k=0,1,3,4,5$. With $h_{j}:=10-2 k_{j}$, the five hyperbinary expansions of $n=10$ are characterized as follows by non-repeating powers of 2 :

- $h_{1}=10$, so $10=\mathbf{8}+\mathbf{2}$

- $h_{2}=8$, so $10=8+1+1$;

- $h_{3}=4$, so $10=4+2+2+1+1$;

- $h_{4}=2$, so $10=4+4+\mathbf{2}$;

- $h_{5}=0$, so $10=4+4+1+1$.

Proof of Theorem 3.5. Comparing Theorem 3.4 with Theorem 2.4, we see that the elements of $\mathcal{P}_{1}(n+1)$ are exactly those of $d_{t}(n-2 k)$ for which $0 \leq k \leq\lfloor n / 2\rfloor$ and $\left(\begin{array}{c}n-k \\ k\end{array}\right)$ is odd. By Definition 3.1 there is a one-to-one correspondence between the powers of $t$ in $d_{t}(n-2 k)$ and the powers of 2 in the binary expansion of $n-2 k$. The result now follows from Theorem 2.4.

4. A 2-parameter extension. Given the similarities between the two types of generalized Stern polynomials defined in $1.2-1.5)$, it is natural to consider the following extension of both types.

Definition 4.1. Let $s$ and $t$ be fixed positive integer parameters. We define the 2-parameter generalized Stern polynomials in the variables $y$ and $z$ by $\omega_{s, t}(0 ; y, z)=0, \omega_{s, t}(1 ; y, z)=1$, and for $n \geq 1$ by

$$
\begin{aligned}
\omega_{s, t}(2 n ; y, z) & =y \omega_{s, t}\left(n ; y^{s}, z^{t}\right), \\
\omega_{s, t}(2 n+1 ; y, z) & =z \omega_{s, t}\left(n ; y^{s}, z^{t}\right)+\omega_{s, t}\left(n+1 ; y^{s}, z^{t}\right) .
\end{aligned}
$$

It is clear from comparing Definitions 4.1 and 1.1 that

$$
\omega_{s, t}(n ; y, 1)=a_{1, s}(n ; y), \quad \omega_{s, t}(n ; 1, z)=a_{2, t}(n ; z) .
$$

The polynomials $\omega_{1,1}(n ; y, z)$ were recently introduced by Mansour [12], in a different notation and as $q$-analogue of the Stern polynomials of Klavžar et al. [11], i.e., of $a_{1,1}(n ; z)$ in our notation. 
The first 16 non-zero polynomials $\omega_{s, t}(n ; y, z)$ are shown in Table 2 .

Table 2. $\omega_{s, t}(n ; y, z)$ for $1 \leq n \leq 16$

\begin{tabular}{ll|rl}
\hline$n$ & $\omega_{s, t}(n ; y, z)$ & $n$ & $\omega_{s, t}(n ; y, z)$ \\
\hline 1 & 1 & 9 & $y^{s^{2}} z^{t}+y^{s+s^{2}} z+z^{t^{2}}+y^{s^{3}}$ \\
2 & $y$ & 10 & $y z^{t^{2}}+y^{1+s^{2}} z^{t}+y^{1+s^{3}}$ \\
3 & $z+y^{s}$ & 11 & $z^{1+t^{2}}+y^{s} z^{t^{2}}+y^{s^{2}} z^{1+t}+y^{s^{3}} z+y^{s+s^{3}}$ \\
4 & $y^{1+s}$ & 12 & $y^{1+s} z^{t^{2}}+y^{1+s+s^{3}}$ \\
5 & $y^{s} z+z^{t}+y^{s^{2}}$ & 13 & $z^{t+t^{2}}+y^{s} z^{1+t^{2}}+y^{s^{3}} z^{t}+y^{s+s^{3}} z+y^{s^{2}+s^{3}}$ \\
6 & $y z^{t}+y^{1+s^{2}}$ & 14 & $y z^{t+t^{2}}+y^{1+s^{3}} z^{t}+y^{1+s^{2}+s^{3}}$ \\
7 & $z^{1+t}+y^{s^{2}} z+y^{s+s^{2}}$ & 15 & $z^{1+t+t^{2}}+y^{s^{3}} z^{1+t}+y^{s^{2}+s^{3}} z+y^{s+s^{2}+s^{3}}$ \\
8 & $y^{1+s+s^{2}}$ & 16 & $y^{1+s+s^{2}+s^{3}}$ \\
\hline
\end{tabular}

4.1. Results. The pairs of Theorems 2.2, 2.4 and 3.2, 3.4 have analogues involving the 2-parameter Stern polynomials. The proofs are similar to those of Theorems 2.4 and 3.4, respectively, and to those of Theorems 2.2 and 3.2, as given in [5]. For this reason, and to avoid repetitive arguments, we leave the details to the interested reader.

We begin by quoting a result by Mansour [12], in different notation, which we will then extend. We adopt the notation $\mathbb{H}_{n}$ from [12] for the set of all hyperbinary expansions of $n$.

Theorem 4.1 ([12, Theorem 3.1]). For any integer $n \geq 1$ we have

$$
\omega_{1,1}(n+1 ; y, z)=\sum_{h \in \mathbb{H}_{n}} y^{p_{h}} z^{q_{h}},
$$

where for a given hyperbinary expansion $h$ of $n$, the exponents $p_{h}$ and $q_{h}$ are the numbers of powers of 2 used exactly once, respectively twice.

EXAmple 7. By Table 2, we have

$$
\omega_{1,1}(11 ; y, z)=z^{2}+y z+y z^{2}+y z+y^{2},
$$

which corresponds, in this order, to the hyperbinary expansions of 10 :

$$
4+4+1+1, \quad 8+1+1, \quad 4+2+2+1+1, \quad 4+4+2, \quad 8+2 .
$$

We see in $\omega_{1,1}(11 ; y, z)$ that there is a repeated monomial. Accordingly, we can rewrite Theorem 4.1 in the following form which is analogous to Theorems 2.1 and 2.3.

COROllary 4.1. Given an integer $n \geq 1$, write

$$
\omega_{1,1}(n+1 ; y, z)=\sum_{j, k \geq 0} c_{j, k}^{(n)} y^{j} z^{k}
$$


Then for each $j, k \geq 0, c_{j, k}^{(n)}$ is the number of hyperbinary expansions of $n$ that have exactly $j$ non-repeated and $k$ repeated powers of 2 .

Returning to Example 7 , we see that $c_{1,1}^{(10)}=2$, which counts the two expansions $8+1+1$ and $4+4+2$.

We are now ready to state the following extension of Theorem 4.1, which can also be seen as a joint extension of Theorems 2.2 and 2.4, and includes an analogue of Theorem 1.1.

THEOREM 4.2. For any integer $n \geq 1$ we have

$$
\omega_{s, t}(n+1 ; y, z)=\sum_{h \in \mathbb{H}_{n}} y^{p_{h}(s)} z^{q_{h}(t)},
$$

where for each hyperbinary expansion $h$ of $n$, the exponents $p_{h}(s), q_{h}(t)$ are polynomials in $s$ and $t$ respectively, with only 0 and 1 as coefficients. Furthermore, if

$$
\begin{array}{cl}
p_{h}(s)=s^{\alpha_{1}}+\cdots+s^{\alpha_{\mu}}, & \mu \geq 0,0 \leq \alpha_{1}<\cdots<\alpha_{\mu}, \\
q_{h}(t)=t^{\beta_{1}}+\cdots+t^{\beta_{\nu}}, & \nu \geq 0,0 \leq \beta_{1}<\cdots<\beta_{\nu},
\end{array}
$$

then the hyperbinary expansion $h \in \mathbb{H}_{n}$ is given by

$$
n=2^{\alpha_{1}}+\cdots+2^{\alpha_{\mu}}+\left(2^{\beta_{1}}+2^{\beta_{1}}\right)+\cdots+\left(2^{\beta_{\nu}}+2^{\beta_{\nu}}\right) .
$$

By convention we assume that $\mu=0$ in 4.7 indicates that $p_{h}(s)$ is the zero polynomial, which in turn means that there is no non-repeated power of 2 in 4.9. Similarly for $\nu=0$ in 4.8 . This theorem also implies that for a given $h \in \mathbb{H}_{n}$ the exponents $\alpha_{j}$ and $\beta_{k}$ in (4.7) and (4.8) are all distinct.

EXAmple 8. By Table 2, we have

$$
\omega_{s, t}(11 ; y, z)=z^{1+t^{2}}+y^{s} z^{t^{2}}+y^{s^{2}} z^{1+t}+y^{s^{3}} z+y^{s+s^{3}} .
$$

If we choose the third term on the right, then according to Theorem 4.2 the corresponding hyperbinary expansion will be $n=4+1+1+2+2$. The other four expansions can be "read off" analogously.

Our next result is an explicit formula, and can be seen as a joint extension of Theorems 3.2 and 3.4. We use again the sequences $d_{t}(n)$ defined in Definition 3.1.

TheOrem 4.3. For integers $s, t \geq 1$ and $n \geq 0$ we have

$$
\omega_{s, t}(n+1 ; y, z)=\sum_{k=0}^{\lfloor n / 2\rfloor}\left(\begin{array}{c}
n-k \\
k
\end{array}\right)^{*} y^{d_{s}(n-2 k)} z^{d_{t}(k)} .
$$

If we set $y=1$, resp. $z=1$, then (4.3) shows that this immediately reduces to Theorems 3.2 and 3.4, respectively. As an easy consequence of Theorem 4.3 we get both Theorem 3.3 and Theorem 3.5 (see the proof of Theorem 3.5). 
By comparing the identity 4.10 with its 1-parameter analogues 3.5 and (3.6), we get the following immediate consequence.

COROLlary 4.2. For an integer $n \geq 1$, the 2-parameter generalized Stern polynomial $\omega_{s, t}(n ; y, z)$ is the discrete convolution of the polynomials $a_{1, s}(n ; y)$ and $a_{2, t}(n ; z)$ if their respective monomials are written in order of increasing exponents.

As an example of this corollary, compare the polynomial in Example 8 with the two entries for $n=11$ in Table 1 .

4.2. Generating functions. We now state a generating function for the 2-parameter generalized Stern polynomials.

TheOREM 4.4. For integers $s, t \geq 1$ we have

$$
x \prod_{j=0}^{\infty}\left(1+y^{s^{j}} x^{2^{j}}+z^{t^{j}} x^{2^{j+1}}\right)=\sum_{n=1}^{\infty} \omega_{s, t}(n ; y, z) x^{n} .
$$

Setting $z=1$, resp. $y=1$, and using the two identities in 4.3 we immediately get the following special cases.

COROLlary 4.3. For integers $t \geq 1$, the generalized Stern polynomials have the following generating functions:

$$
\begin{aligned}
& x \prod_{j=0}^{\infty}\left(1+z^{t^{j}} x^{2^{j}}+x^{2^{j+1}}\right)=\sum_{n=1}^{\infty} a_{1, t}(n ; z) x^{n}, \\
& x \prod_{j=0}^{\infty}\left(1+x^{2^{j}}+z^{t^{j}} x^{2^{j+1}}\right)=\sum_{n=1}^{\infty} a_{2, t}(n ; z) x^{n} .
\end{aligned}
$$

Various cases of (4.11)-4.13 are already known: 4.13) was proved in [5], with the subcases $t=1$ and $t=2$ earlier obtained in 1 and [8], respectively. The case $s=t=1$ of 4.11 was more recently proved in [12, with the subcase $z=1$ earlier obtained in [20]. Finally, the analogue for Stern's diatomic sequence, i.e., the case $y=z=1$ in (4.11), goes back to Carlitz [4].

The proof of 4.11 is very similar to that of 4.13 ), given as Proposition 5.1 in [5, and the inclusion of a second parameter and a second variable presents no additional complication. We therefore leave the details to the reader.

4.3. A single-variable analogue. Finally in this section, we note that working with two variables $y$ and $z$ is, in most of this section, not strictly necessary. We therefore define the single-variable analogue of $\omega_{s, t}(n ; y, z)$ by

$$
\omega_{s, t}(n ; z):=\omega_{s, t}(n ; z, z),
$$

i.e., by setting $y=z$. The sequence $\omega_{s, t}(n ; z)$ then has an obvious generating function coming from 4.11, and the identity 4.10 gives the explicit 
formula

$$
\omega_{s, t}(n+1 ; z)=\sum_{k=0}^{\lfloor n / 2\rfloor}\left(\begin{array}{c}
n-k \\
k
\end{array}\right)^{*} z^{d_{s}(n-2 k)+d_{t}(k)} .
$$

Only for the recurrence (4.1), 4.2 do we need to retain both variables $y$ and $z$.

It turns out that no information is lost by setting $y=z$. Indeed, it is clear from (4.1) and (4.2) that all exponent polynomials in $s$ have base $y$, and those in $t$ have base $z$ (see also Table 2). Only the summand 1 in the exponent may be ambiguous. However, it follows from (4.1) and 4.2 that for even $n$, any 1 that occurs is part of a polynomial $p_{h}(s)$, and for odd $n$, any 1 that occurs belongs to a polynomial $q_{h}(t)$.

With a small adjustment to this effect, Theorem 4.2 could then be stated with the polynomial $\omega_{s, t}(n+1 ; z)$ on the left-hand side of 4.6$)$. For instance, with

$$
\omega_{s, t}(11 ; z)=z^{1+t^{2}}+z^{s+t^{2}}+z^{1+t+s^{2}}+z^{1+s^{3}}+z^{s+s^{3}},
$$

we can still "read off" the hyperbinary expansions of 10, as we did in Example 8.

However, for Theorem 4.1 and Corollary 4.1 to be meaningful, we require the variables $y$ and $z$ to be kept separate.

\section{Some further remarks}

5.1. The strength and usefulness of Theorems 2.2 and 2.4 stem from the fact that, given a generalized Stern polynomial $a_{1, t}(n+1 ; z)$ or $a_{2, t}(n+1 ; z)$, we can use the exponents of $z$, which are polynomials in $t$, to fully characterize all hyperbinary expansions of $n$. Given the forms 2.2 and 2.4 of these exponents, it becomes clear that we can fix an integer $t \geq 2$ and consider the resulting polynomials with numerical, rather than polynomial, exponents of $z$.

In particular, if we take $t=2$, then $a_{2,2}(n+1 ; z)$ is just the Stern polynomial as first introduced in [8]. It is quite easy to compute, as is the type-1 analogue $a_{1,2}(n+1 ; z)$. Using Theorems 2.2 and 2.4 and the uniqueness of a binary expansion, we get the following consequence.

COROllary 5.1. For $n \geq 1$, let

$$
a_{1,2}(n+1 ; z)=\sum_{j \geq 1} z^{p_{j}}, \quad a_{2,2}(n+1 ; z)=\sum_{j \geq 1} z^{q_{j}} .
$$

Then each hyperbinary expansion of $n$

(1) corresponds to exactly one exponent $p_{j}$ as follows: if $p_{j}=2^{\alpha_{1}}+$ $\cdots+2^{\alpha_{r}} \geq 1$ is the binary expansion of $p_{j}$, then exactly the powers 
$2^{\alpha_{1}}, \ldots, 2^{\alpha_{r}}$ are not repeated in the hyperbinary expansion, with $p_{j}=0$ corresponding to the expansion that has only repeated powers of 2;

(2) corresponds to exactly one exponent $q_{j}$ as follows: if $p_{j}=2^{\beta_{1}}+$ $\cdots+2^{\beta_{s}} \geq 1$ is the binary expansion of $q_{j}$, then exactly the powers $2^{\beta_{1}}, \ldots, 2^{\beta_{s}}$ are repeated in the hyperbinary expansion, with $q_{j}=0$ corresponding to the unique binary expansion of $n$.

EXAmple 9 . Let $n=12$ and consider $a_{2,2}(13 ; z)=1+z+z^{2}+z^{5}+z^{6}$ (see Table 1 ). Then the exponents have binary expansions $0,1,2,1+4$, and $2+4$. Accordingly, the hyperbinary expansions of 12 are

$$
4+8, \quad \mathbf{1}+\mathbf{1}+2+8, \quad \mathbf{2}+\mathbf{2}+8, \quad \mathbf{1}+\mathbf{1}+2+\mathbf{4}+\mathbf{4}, \quad \mathbf{2}+\mathbf{2}+\mathbf{4}+\mathbf{4}
$$

The non-repeated parts (not in boldface) are just binary expansions of the differences between $n=12$ and the sums of the repeated parts (if any).

On the other hand, we have $a_{1,2}(13 ; z)=1+z^{2}+z^{8}+z^{10}+z^{12}$, and the exponents have binary expansions $0,2,8,2+8$, and $4+8$. As expected, these correspond to the non-boldface parts in the hyperbinary expansions above.

5.2. The 2-parameter polynomials $\omega_{2,2}(n ; y, z)$, together with a modified version of Theorem 4.2, can also be used to obtain complete characterizations of all hyperbinary expansions. If, in addition, we set $y=z$, then the recurrence relations 4.1) and 4.2) simplify to

$$
\begin{aligned}
\omega_{2,2}(2 n ; z) & =z \omega_{2,2}\left(n ; z^{2}\right), \\
\omega_{2,2}(2 n+1 ; z) & =z \omega_{2,2}\left(n ; z^{2}\right)+\omega_{2,2}\left(n+1 ; z^{2}\right),
\end{aligned}
$$

which gives rise to the polynomial sequence $1, z, z+z^{2}, z^{3}, z^{2}+z^{3}+z^{4}$, $z^{3}+z^{5}$, etc., where the degree is easy to verify as $\operatorname{deg}\left(\omega_{2,2}(n ; z)\right)=n-1$.

From 4.15 we immediately get the explicit formula

$$
\omega_{2,2}(n+1 ; z)=\sum_{k=0}^{\lfloor n / 2\rfloor}\left(\begin{array}{c}
n-k \\
k
\end{array}\right)^{*} z^{n-k} .
$$

By Theorem 4.2 with $s=t=2$ the parts in each of the hyperbinary expansions of $n$ are given by the binary expansion of each of the exponents in the polynomial $\omega_{2,2}(n+1 ; z)$.

EXAMPLE 10. From the recurrence relations or from (5.1) we get $\omega_{2,2}(13 ; z)=z^{6}+z^{7}+z^{10}+z^{11}+z^{12}$ (compare also with Table 2 ). Then the parts in each of the five hyperbinary expansions are given by the binary expansions of the exponents, namely $2+4,1+2+4,2+8,1+2+8$, and $4+8$, which is consistent with Example 9 .

To continue with the argument before this last example, we note that by using (5.1) we can combine this observation with Theorems 3.3 and 3.5 to obtain the following result, which may serve as a summary. 
COROLlary 5.2. Let $n \geq 2$ be an integer and denote by $k_{1}, \ldots, k_{\nu}$ those integers $0 \leq k \leq\lfloor n / 2\rfloor$ for which $\left(\begin{array}{c}n-k \\ k\end{array}\right)$ is odd. Then each hyperbinary expansion (HBE) of $n$ corresponds to exactly one $k_{j}, j=1, \ldots, \nu$, as follows: the powers of 2 in the binary expansion

(i) of $k_{j}$ are the repeated powers of 2 in the HBE of $n$;

(ii) of $n-2 k_{j}$ are the non-repeated powers of 2 in the HBE of $n$;

(iii) of $n-k_{j}$ are the powers of 2 that occur in the HBE of $n$.

5.3. The sequences studied in this paper can be further generalized if we first observe that the various generating functions (4.11)-4.13) can be used to define the respective polynomial sequences. The idea now is to use an arbitrary positive integer sequence in place of the sequence $\left(t^{j}\right)_{j \geq 0}$ that occurs as an exponent of the variable $z$. While we might expect a large number of different classes of generalized Stern polynomials, we will see that they can all essentially be reduced to the three types of generalized Stern polynomials considered in this paper. We only deal with one type here, and leave the others to the interested reader.

DeFinition 5.1. Let $\sigma$ be a positive integer sequence given by $\left(\sigma_{j}\right)_{j \geq 0}$. We define the type-1 $\sigma$-generalized Stern polynomials $a_{1, \sigma}(n ; z)$ by the generating function

$$
x \prod_{j=0}^{\infty}\left(1+z^{\sigma_{j}} x^{2^{j}}+x^{2^{j+1}}\right)=\sum_{n=1}^{\infty} a_{1, \sigma}(n ; z) x^{n} .
$$

In addition, we set $a_{1, \sigma}(0 ; z)=0$.

Just as the generating functions $(4.12)$ and $(4.13)$ imply the recurrence relations $1.2-1.5$, we can use 5.2 to obtain the following recurrence relations.

TheOREM 5.1. Let $\sigma=\left(\sigma_{j}\right)_{j \geq 0}$ be a positive integer sequence. With $a_{1, \sigma}(1 ; z)=1$ and for $n \geq 1$ we have

$$
\begin{aligned}
a_{1, \sigma}(2 n ; z) & =z^{\sigma_{0}} a_{1, \sigma}\left(n ; z^{+}\right), \\
a_{1, \sigma}(2 n+1 ; z) & =a_{1, \sigma}\left(n ; z^{+}\right)+a_{1, \sigma}\left(n+1 ; z^{+}\right),
\end{aligned}
$$

where $z^{+}$indicates that the following sequence term must be taken in the exponent, i.e., $\left(z^{\sigma_{j}}\right)^{+}=z^{\sigma_{j+1}}$.

The proof is based on isolating the first factor in the product in (5.2), manipulating the resulting products and series, and equating coefficients of like powers of $z$. We leave the details to the reader. As an illustration, we use Theorem 5.1 to compute the first few polynomials $a_{1, \sigma}(n ; z)$ for a general sequence $\sigma$. 
ExAmPle 11. Since $a_{1, \sigma}(1 ; z)=1$, 5.3 gives $a_{1, \sigma}(2 ; z)=z^{\sigma_{0}}$. From (5.4) with $n=1$ we then get

$$
a_{1, \sigma}(3 ; z)=a_{1, \sigma}\left(1 ; z^{+}\right)+a_{1, \sigma}\left(2 ; z^{+}\right)=1+\left(z^{\sigma_{0}}\right)^{+}=1+z^{\sigma_{1}} .
$$

Next, (5.3) and (5.4) with $n=2$ give

$$
\begin{aligned}
a_{1, \sigma}(4 ; z) & =z^{\sigma_{0}} a_{1, \sigma}\left(2 ; z^{+}\right)=z^{\sigma_{0}}\left(z^{\sigma_{0}}\right)^{+}=z^{\sigma_{0}+\sigma_{1}} \\
a_{1, \sigma}(5 ; z) & =a_{1, \sigma}\left(2 ; z^{+}\right)+a_{1, \sigma}\left(3 ; z^{+}\right)=\left(z^{\sigma_{0}}\right)^{+}+\left(1+\left(z^{\sigma_{1}}\right)^{+}\right) \\
& =1+z^{\sigma_{1}}+z^{\sigma_{2}}
\end{aligned}
$$

and 5.3 with $n=3$,

$$
a_{1, \sigma}(6 ; z)=z^{\sigma_{0}} a_{1, \sigma}\left(3 ; z^{+}\right)=z^{\sigma_{0}}\left(1+\left(z^{\sigma_{1}}\right)^{+}\right)=z^{\sigma_{0}}+z^{\sigma_{0}+\sigma_{2}} .
$$

Finally, to jump ahead, we use (5.4) to compute the case of our standard example

$$
\begin{aligned}
a_{1, \sigma}(11 ; z) & =\left(1+\left(z^{\sigma_{1}}\right)^{+}+\left(z^{\sigma_{2}}\right)^{+}\right)+\left(\left(z^{\sigma_{0}}\right)^{+}+\left(z^{\sigma_{0}+\sigma_{2}}\right)^{+}\right) \\
& =1+z^{\sigma_{1}}+z^{\sigma_{2}}+z^{\sigma_{3}}+z^{\sigma_{1}+\sigma_{3}} .
\end{aligned}
$$

If we compare the polynomial in 5.5 with the entry for $a_{1, t}(11 ; z)$ in Table 1, we see that there is a one-to-one correspondence between a power $t^{j}$ and a term $\sigma_{j}$ of the defining sequence. This is not surprising and follows from Theorem 5.1, if we compare it with Definition 1.1. This correspondence also means that most results in Sections 2 and 3 carry over to the $\sigma$-generalized case.

5.4. Finally we mention an interesting connection with Chebyshev polynomials of the second kind, $U_{n}(x)$. Using a well-known explicit formula (see, e.g., [15, p. 39]), it is easy to see that

$$
\sum_{k=0}^{\lfloor n / 2\rfloor}\left(\begin{array}{c}
n-k \\
k
\end{array}\right) z^{n-k}=(-i \sqrt{z})^{n} U_{n}\left(\frac{i}{2} \sqrt{z}\right),
$$

and (5.1) immediately implies

$$
\omega_{2,2}(n+1 ; z) \equiv(-i \sqrt{z})^{n} U_{n}\left(\frac{i}{2} \sqrt{z}\right)(\bmod 2),
$$

where the congruence is to be seen as a congruence for each coefficient. Since the left-hand side has only 0 and 1 as coefficients, this means that $\omega_{2,2}(n+1 ; z)$ can be completely determined from the Chebyshev polynomials of the second kind.

A similar congruence was obtained in [8, Proposition 6.1] for the Stern polynomials $a_{2,2}(n+1 ; z)$.

Acknowledgments. Research supported in part by the Natural Sciences and Engineering Research Council of Canada, Grant \# 145628481. 


\section{References}

[1] B. Bates and T. Mansour, The q-Calkin-Wilf tree, J. Combin. Theory Ser. A 118 (2011), 1143-1151.

[2] N. Calkin and H. S. Wilf, Recounting the rationals, Amer. Math. Monthly 107 (2000), 360-363.

[3] L. Carlitz, Single variable Bell polynomials, Collect. Math. 14 (1960), 13-25.

[4] L. Carlitz, A problem in partitions related to the Stirling numbers, Bull. Amer. Math. Soc. 70 (1964), 275-278.

[5] K. Dilcher and L. Ericksen, Hyperbinary expansions and Stern polynomials, Electron. J. Combin. 22 (2015), no. 2, paper 2.24, 18 pp.

[6] K. Dilcher and L. Ericksen, Some tilings, colorings and lattice paths via Stern polynomials, preprint, 2015.

[7] K. Dilcher and L. Ericksen, Continued fractions and Stern polynomials, Ramanujan J., online (2017); doi:10.1007/s11139-016-9864-3.

[8] K. Dilcher and K. B. Stolarsky, A polynomial analogue to the Stern sequence, Int. J. Number Theory 3 (2007), 85-103.

[9] M. Gawron, A note on the arithmetic properties of Stern polynomials, Publ. Math. Debrecen 85 (2014), 453-465.

[10] C. Giuli and R. Giuli, A primer on Stern's diatomic sequence, Part III: Additional results, Fibonacci Quart. 17 (1979), 318-320.

[11] S. Klavžar, U. Milutinović, and C. Petr, Stern polynomials, Adv. Appl. Math. 39 (2007), 86-95.

[12] T. Mansour, q-Stern polynomials as numerators of continued fractions, Bull. Polish Acad. Sci. Math. 63 (2015), 11-18.

[13] OEIS Foundation Inc., The On-Line Encyclopedia of Integer Sequences, 2011, http: //oeis.org

[14] B. Reznick, Some binary partition functions, in: Analytic Number Theory: Proceedings of a Conference in Honor of Paul T. Bateman (B. C. Berndt et al., eds.), Birkhäuser, Boston, 1990, 451-477.

[15] T. J. Rivlin, Chebyshev Polynomials, 2nd ed., Wiley, New York, 1990.

[16] A. Schinzel, On the factors of Stern polynomials (remarks on the preceding paper of M. Ulas), Publ. Math. Debrecen 79 (2011), 83-88.

[17] A. Schinzel, Stern polynomials as numerators of continued fractions, Bull. Polish Acad. Sci. Math. 62 (2014), 23-27.

[18] A. Schinzel, The leading coefficients of Stern polynomials, in: From Arithmetic to Zeta-Functions: Number Theory in Memory of Wolfgang Schwarz (J. Sander et al., eds.), Springer, 2016, 427-434.

[19] R. P. Stanley and H. S. Wilf, Refining the Stern diatomic sequence, preprint, 2010, http://www-math.mit.edu/ rstan/papers/stern.pdf

[20] M. Ulas, On certain arithmetic properties of Stern polynomials, Publ. Math. Debrecen 79 (2011), 55-81.

[21] M. Ulas, Arithmetic properties of the sequence of degrees of Stern polynomials and related results, Int. J. Number Theory 8 (2012), 669-687.

Karl Dilcher

Department of Mathematics and Statistics

Dalhousie University

Halifax, Nova Scotia, B3H 4R2, Canada

E-mail: dilcher@mathstat.dal.ca
Larry Ericksen

P.O. Box 172

Millville, NJ 08332-0172, U.S.A.

E-mail: LE22@cornell.edu 